\title{
SPECTRA OF WEIGHTED COMPOSITION OPERATORS ON WEIGHTED BANACH SPACES OF ANALYTIC FUNCTIONS
}

\author{
R. Aron And M. LindströM
}

\begin{abstract}
We determine the spectra of weighted composition operators acting on the weighted Banach spaces of analytic functions $H_{v_{p}}^{\infty}$ when the symbol $\phi$ has a fixed point in the open unit disk. Further, we apply this result to give the spectra of composition operators on Bloch type spaces. In particular, we answer in the affirmative a conjecture by MacCluer and Saxe.
\end{abstract}

\section{Introduction.}

We denote by $H(D)$ the space of holomorphic functions on the open unit disk $D$ and consider the weighted Bergman spaces of infinite order

$$
H_{v}^{\infty}=\left\{f \in H(D):|| f \|_{v}:=\sup _{z \in D} v(z)|f(z)|<\infty\right\}
$$

and

$$
H_{v}^{0}=\left\{f \in H_{v}^{\infty}: \lim _{|z| \rightarrow 1} v(z)|f(z)|=0\right\},
$$

endowed with the norm $\|\cdot\|_{v}$, where the weight function $v: D \rightarrow \mathbb{R}_{+}$is radial, continuous, nonincreasing with respect to $|z|$ and satisfy $\lim _{|z| \rightarrow 1} v(z)=0$. The associated weight $\tilde{v}$ is defined by

$$
\tilde{v}(z)=\left(\sup \left\{|f(z)|: f \in H_{v}^{\infty},\|f\|_{v} \leq 1\right\}\right)^{-1} .
$$

Then $\tilde{v}$ is also a weight. If we take $\tilde{v}$ instead of $v$, both the spaces $H_{v}^{\infty}$ and $H_{v}^{0}$ and the norm $\|\cdot\|_{v}$ do not change. Further, given $z \in D$, the element $\delta_{z} \in\left(H_{v}^{\infty}\right)^{*}$ defined by $\delta_{z}(f)=f(z)$ satisfies $\left\|\delta_{z}\right\|_{v}=1 / \tilde{v}(z)$, and for each $z \in D$ there is $f_{z} \in H_{v}^{\infty},\left\|f_{z}\right\|_{v} \leq 1$, such that $\left|f_{z}(z)\right|=1 / \tilde{v}(z)$. These remarks and more about the associated weight $\tilde{v}$ can be found in $[\mathrm{BBT}]$. In this paper we are interested in the Bergman spaces of infinite order for the weights $v_{p}(z)=\left(1-|z|^{2}\right)^{p}, p>0$, on $D$. Let us point out that $\tilde{v}_{p}(z)=v_{p}(z)$ and notice also that the norm $\|\cdot\|_{v_{p}}$ is finer than the compact-open topology. Recall that the polynomials are dense in $H_{v_{p}}^{0}$ and

1991 Mathematics Subject Classification. Primary 47B38 Secondary 46E15.

Key words and phrases. Weighted Bergman spaces of infinite order, weighted composition operator, spectrum, essential norm, essential spectral radius.

The research of the second author was partially supported by the Academy of Finland Project No. 51906; the research of this paper was carried out while this author was visiting Kent State University, whose hospitality is acknowledged with thanks. 
therefore contained in $H_{v_{p}}^{\infty}$ and that each function in $H_{v_{p}}^{\infty}$ is the weak* limit of a sequence of polynomials.

When restricted to various Banach spaces of analytic functions the operation of composition with $\phi$, usually denoted $C_{\phi}$ and $\phi$ is a self map on the open unit disk in the complex plane, has been object for intense study in recent years, especially the problem of relating operator-theoretic properties of $C_{\phi}$ to function theoretic properties of $\phi$. See the books of Cowen and MacCluer [CM] and Shapiro [Sh] for discussions of composition operators on classical spaces of holomorphic functions.

To characterize the spectrum $\sigma\left(C_{\phi}\right)$ of a composition operator acting on Banach spaces of analytic functions when $C_{\phi}$ is a non-compact operator has recently been the object of investigations in [CM1], [Z] and [MS]. More precisely, MacCluer and Saxe [MS] have characterized the spectrum of $C_{\phi}$ on the Bergman spaces $A^{p}$ and Hardy spaces $H^{p}, 1 \leq p<\infty$, when $\phi$ is univalent, not an automorphism, which fixes a point in $D$. Cowen and MacCluer [CM1] had earlier given $\sigma_{A^{2}}\left(C_{\phi}\right)$ and $\sigma_{H^{2}}\left(C_{\phi}\right)$ for such $\phi$. Kamowitz [K1] was the first to study the spectrum of $C_{\phi}$ on $H^{p}, 1 \leq p<\infty$, when $\phi$ is not inner and has a fixed point in $D$. Further, L. Zheng [Z] has recently shown that $\sigma_{H^{\infty}}\left(C_{\phi}\right)=\bar{D}$, when $\phi$, not an automorphism, fixes a point of $D$ and $r_{e, H^{\infty}}\left(C_{\phi}\right) \neq 0$. All the proofs of the above mentioned results follow the same pattern and we shall also make use of this method of proof in our investigation of the spectrum of the bounded weighted composition operator $C_{\psi, \phi}: H_{v_{p}}^{\infty} \rightarrow H_{v_{p}}^{\infty}, 0<p<\infty$. Since we are dealing with bounded weighted composition operators $C_{\psi, \phi}$ which can be non-compact the spectrum $\sigma_{H_{v_{p}}^{\infty}}\left(C_{\psi, \phi}\right)$ will be given in terms of the essential spectral radius $r_{e, H_{v_{p}}^{\infty}}\left(C_{\psi, \phi}\right)$. As an application of the main result we determine the spectrum of a bounded composition operator $C_{\phi}$ on the Bloch type space $\mathcal{B}_{p}, 0<p<\infty$, when $\phi$, not an automorphism, fixes a point in $D$. If $\phi$, in addition, is univalent and $r_{e, H^{2}}\left(C_{\phi}\right) \neq 0$, then it follows that the spectrum of $C_{\phi}$ on the Bloch space coincides with $\bar{D}$, which answers in the affirmative a conjecture by MacCluer and Saxe [MS].

The weighted composition operator $C_{\psi, \phi}$ is defined by $C_{\psi, \phi}(f)=\psi(f \circ \phi)$, where $\psi, \phi \in H(D)$ are non-trivial and $\phi(D) \subset D$. By Proposition 3.1 in [CHD] such operators $C_{\psi, \phi}$ are bounded on $H_{v_{p}}^{\infty}$ if and only if

$$
\sup _{z \in D} \frac{|\psi(z)|\left(1-|z|^{2}\right)^{p}}{\left(1-|\phi(z)|^{2}\right)^{p}}<\infty .
$$

See also [MR] for a similar result. If $\psi(z)=1$, then $C_{\psi, \phi}=C_{\phi}$ is always bounded (see Theorem 2.3 in [BDLT]). Hence $C_{\psi, \phi}$ is bounded for $\psi \in H^{\infty}$. For $a \in D$ and $\phi_{a}(z)=\frac{a-z}{1-\bar{a} z}$ the composition operator $C_{\phi_{a}}$ on $H_{v_{p}}^{\infty}$ is an isomorphism and $C_{\phi_{a}}^{-1}=C_{\phi_{a}}$. In the case $\psi=\phi^{\prime}$ and $1 \leq p<\infty$, then by the Schwarz-Pick lemma $\left|\phi^{\prime}(z)\right| \leq\left(1-|\phi(z)|^{2}\right) /\left(1-|z|^{2}\right)$. Thus we conclude by Theorem 2.3 in [BDLT] that $C_{\phi^{\prime}, \phi}$ is bounded. If $\left\|C_{\psi, \phi}\right\|_{e, v_{p}}$ denotes the essential norm of $C_{\psi, \phi}$, i.e., the distance from the compact operators,

$$
\left\|C_{\psi, \phi}\right\|_{e, v_{p}}=\inf \left\{\left\|C_{\psi, \phi}-K\right\|: K \text { is a compact operator on } H_{v_{p}}^{\infty}\right\},
$$

then we get from $[\mathrm{CHD}]$ or $[\mathrm{MR}]$ (see also $[\mathrm{BDL}]$ ) that

$$
\left\|C_{\psi, \phi}\right\|_{e, v_{p}}=\lim _{r \rightarrow 1} \sup _{|\phi(z)|>r} \frac{|\psi(z)|\left(1-|z|^{2}\right)^{p}}{\left(1-|\phi(z)|^{2}\right)^{p}} .
$$


Following Shields-Williams $[\mathrm{SW}]$ we consider the pairing

$$
(f, g)=\int_{D} f(z) g(\bar{z})\left(1-|z|^{2}\right)^{p} d A(z), \quad f \in H_{v_{p}}^{\infty}, g \in A^{1}
$$

where $d A$ is normalized Lebesgue measure on $D$ and the Bergman space $A^{1}$ is the dual of $H_{v_{p}}^{0}$ and $H_{v_{p}}^{\infty}$ is the bidual of $H_{v_{p}}^{0}$. The kernel

$$
K_{w}(z)=\frac{p+1}{(1-w z)^{2+p}}=\sum_{n=0}^{\infty} \frac{(p+1) \ldots(p+n+1)}{n !}(z w)^{n}, \quad z, w \in D
$$

is in both $H_{v_{p}}^{0}$ and $A^{1}$ and reproduces $f(w)=\left(f, K_{w}\right)$ for all $f \in H_{v_{p}}^{\infty}$.

For $v$ an arbitrary weight let $H_{v, m}^{\infty}:=z^{m} H_{v}^{\infty}$ be the subspace of $H_{v}^{\infty}$. For the weight $v_{p}$ we shall below show that it is a Banach space and that $H_{v_{p}, m}^{\infty}$ is equivalently described as $\left\{f \in H_{v_{p}}^{\infty}: f\right.$ has a zero of at least order $\mathrm{m}$ at zero $\}$.

\section{The main result.}

The proof of our main result, Theorem 7, is based on Proposition 1 which is a crucial result in our investigation.

Proposition 1. Let $p>0$ and $m \in \mathbb{N}, m \geq 1$. Then there exists a constant $c(m, p)$ depending only on $m$ and $p$, so that if $f(z)=\sum_{n=m}^{\infty} a_{n} z^{n} \in H_{v_{p}}^{\infty}$ and $w \in D$, then

$$
|f(w)| \leq c(m, p) \frac{|w|^{m}}{\left(1-|w|^{2}\right)^{p}}\|f\|_{v_{p}}
$$

Before we prove Proposition 1 we need the following easily proved result.

Sublemma 2. Let $\alpha \in \mathbb{R}$ and $m \in \mathbb{N}, m \geq 1$. Then

$$
\sum_{k=0}^{m}\left(\begin{array}{c}
-\alpha \\
k
\end{array}\right)\left(\begin{array}{c}
\alpha \\
m-k
\end{array}\right)=0
$$

Proof. For $\alpha$ any real number and $|z|<1,(1+z)^{\alpha}=\sum_{m=0}^{\infty}\left(\begin{array}{c}\alpha \\ m\end{array}\right) z^{m}$. Thus the Cauchy product gives

$$
1=(1+z)^{\alpha}(1+z)^{-\alpha}=\sum_{m=0}^{\infty}\left(\sum_{k=0}^{m}\left(\begin{array}{c}
-\alpha \\
k
\end{array}\right)\left(\begin{array}{c}
\alpha \\
m-k
\end{array}\right)\right) z^{m}
$$

and therefore $\sum_{k=0}^{m}\left(\begin{array}{c}-\alpha \\ k\end{array}\right)\left(\begin{array}{c}\alpha \\ m-k\end{array}\right)=0$ for any $m \in \mathbb{N}, m \geq 1$.

Proof of Proposition 1. Let $f(z)=\sum_{n=m}^{\infty} a_{n} z^{n}$. By the Remark after Lemma 9 in $[\mathrm{SW}]$,

$$
f(w)=\left(f, K_{w}\right)=\lim _{r \rightarrow 1}\left(f_{r}, K_{w}\right)=\lim _{r \rightarrow 1} \sum_{n=m}^{\infty} \frac{a_{n} c_{n}(p)(r w)^{n} n !}{(p+1) \ldots(p+n+1)}=\left(f, K_{w}^{m}\right),
$$


where $K_{w}^{m}(z)=\sum_{n=m}^{\infty} c_{n}(p)(z w)^{n}$ and $c_{n}(p):=(-1)^{n}(p+1)\left(\begin{array}{c}-2-p \\ n\end{array}\right)$ for $n \geq 0$. Hence

$$
|f(w)|=\left|\int_{D} f(z) K_{w}^{m}(\bar{z})\left(1-|z|^{2}\right)^{p} d A(z)\right| \leq\|f\|_{v_{p}} \int_{D}\left|K_{w}^{m}(\bar{z})\right| d A(z) .
$$

Let now $z, w \in D$ be fixed. Then

$$
(1-z w)^{2+p} K_{w}^{m}(z)=(p+1)-(1-z w)^{2+p}\left\{c_{0}(p)+c_{1}(p)(z w)+. .+c_{m-1}(p)(z w)^{m-1}\right\}
$$

Since $(1-z w)^{2+p}=\sum_{k=0}^{\infty}\left(\begin{array}{c}2+p \\ k\end{array}\right)(-z w)^{k}$ and $\sum_{k=0}^{m-1}\left(\begin{array}{c}-2-p \\ k\end{array}\right)\left(\begin{array}{c}2+p \\ m-1-k\end{array}\right)=0$ for $m \in \mathbb{N}$, $m \geq 2$ by Sublemma 2, it follows that

$$
\begin{gathered}
|1-z w|^{2+p}\left|K_{w}^{m}(z)\right| \leq|z w|^{m}\left\{c_{0}(p) \sum_{k=m}^{\infty}\left|\left(\begin{array}{c}
2+p \\
k
\end{array}\right)\right||z w|^{k-m}+\right. \\
\left.c_{1}(p) \sum_{k=m-1}^{\infty}\left|\left(\begin{array}{c}
2+p \\
k
\end{array}\right)\right||z w|^{k-m+1}+\ldots+c_{m-1}(p) \sum_{k=1}^{\infty}\left|\left(\begin{array}{c}
2+p \\
k
\end{array}\right)\right||z w|^{k-1}\right\} \\
\leq|w|^{m}\left\{c_{0}(p)+c_{1}(p)+\ldots+c_{m-1}(p)\right\} \sum_{k=1}^{\infty}\left|\left(\begin{array}{c}
2+p \\
k
\end{array}\right)\right| .
\end{gathered}
$$

Next, we obtain that

$$
\begin{aligned}
& \left|\left(\begin{array}{c}
2+p \\
k
\end{array}\right)\right|=\left|\frac{(2+p)(1+p) \ldots(3+p-k)}{k !}\right|=\exp \left\{\sum_{j=1}^{k} \log \left|1-\frac{3+p}{j}\right|\right\} \\
= & \exp \left\{\sum_{j=1}^{k}\left(\frac{-3-p}{j}+O\left(\frac{1}{j^{2}}\right)\right)\right\}=\exp \{(-3-p) \log (k)+O(1)\}=O\left(k^{-3-p}\right),
\end{aligned}
$$

so there is a constant $c(m, p)$ only depending on $m$ and $p$ such that

$$
|1-z w|^{2+p}\left|K_{w}^{m}(z)\right| \leq|w|^{m} c(m, p) .
$$

Consequently,

$$
|f(w)| \leq\|f\|_{v_{p}} c(m, p)|w|^{m} \int_{D} \frac{1}{|1-\bar{z} w|^{2+p}} d A(z)
$$

Finally by Lemma 10, p.87 in [W],

$$
\int_{D} \frac{1}{|1-\bar{z} w|^{2+p}} d A(z) \leq c(p) \frac{1}{\left(1-|w|^{2}\right)^{p}}
$$

and the estimate follows. 
Lemma 3. Let $w \in D$ and $|w| \geq 1 / 2$. Then

$$
\left\|\delta_{w}\right\|_{v, m} \leq\left\|\delta_{w}\right\|_{v} \leq 2^{m}\left\|\delta_{w}\right\|_{v, m} .
$$

Proof. Since $H_{v, m}^{\infty} \subset H_{v}^{\infty}$, it follows that $\left\|\delta_{w}\right\|_{v, m} \leq\left\|\delta_{w}\right\|_{v}$. For fixed $w \in D$ there is a $f_{w} \in H_{v}^{\infty},\left\|f_{w}\right\|_{v} \leq 1$, such that $\left|f_{w}(w)\right|=1 / \tilde{v}(w)$. Let $g_{w}(z):=z^{m} f_{w}(z)$. Then $\left\|g_{w}\right\|_{v} \leq 1$ and

$$
|| \delta_{w}||_{v, m} \geq\left|g_{w}(w)\right|=|w|^{m}\left|f_{w}(w)\right| \geq 1 / 2^{m} 1 / \tilde{v}(w)=1 / 2^{m}\left\|\delta_{w}\right\|_{v} .
$$

Lemma 4. Let $p>0, m \in \mathbb{N}, m \geq 1$ and suppose that the operator $C_{\psi, \phi}: H_{v_{p}}^{\infty} \rightarrow$ $H_{v_{p}}^{\infty}$ is bounded. If $\phi(0)=0$, then $H_{v_{p}, m}^{\infty}$ is invariant under $C_{\psi, \phi}$, a closed subspace of $H_{v_{p}}^{\infty}$ and $\sigma_{H_{v_{p}, m}^{\infty}}\left(C_{\psi, \phi}\right) \subset \sigma_{H_{v_{p}}^{\infty}}\left(C_{\psi, \phi}\right)$.

Proof. Since $\phi(z)=z g(z)$, where $g \in H^{\infty}$, it follows for $f \in H_{v_{p}}^{\infty}$ that $C_{\psi, \phi}\left(z^{m} f\right)=$ $z^{m} g^{m} C_{\psi, \phi}(f)$. Therefore $H_{v_{p}, m}^{\infty}$ is an invariant subspace under $C_{\psi, \phi}$.

Now we have

$$
H_{v_{p}, m}^{\infty}=\left\{f \in H_{v_{p}}^{\infty}: f \text { has a zero of at least order } \mathrm{m} \text { at zero }\right\} .
$$

Indeed, let $f(z)=\sum_{n=m}^{\infty} a_{n} z^{n} \in H_{v_{p}}^{\infty}$. Then we write $f(z)=z^{m} \sum_{n=0}^{\infty} a_{m+n} z^{n}$ and Proposition 1 implies that there is a constant $c(m, p)$ such that for all $z \in D$,

$$
|z|^{m}\left|\sum_{n=0}^{\infty} a_{m+n} z^{n}\right| v_{p}(z) \leq c(m, p)|z|^{m}|| f \|_{v_{p}} .
$$

Thus $f \in H_{v_{p}, m}^{\infty}$. The other inclusion is obvious.

Next we notice that $H_{v_{p}, m}^{\infty}$ is a closed subspace of $H_{v_{p}}^{\infty}$. Take a sequence $z^{m} f_{j}(z) \in H_{v_{p}, m}^{\infty}$ such that $z^{m} f_{j}(z) \rightarrow g(z) \in H_{v_{p}}^{\infty}$ in the norm $\|\cdot\|_{v_{p}}$. Then $z^{m} f_{j}(z) \rightarrow g(z)$ with respect to the compact-open topology and we get that $g(z)=z^{m} h(z)$, where $h(z) \in H(D)$. By the above description of $H_{v_{p}, m}^{\infty}$ we conclude that $g \in H_{v_{p}, m}^{\infty}$.

From this description we also conclude that $H_{v_{p}, m}^{\infty}$ has finite codimension in $H_{v_{p}}^{\infty}$. Because a modification of the proof of Lemma 7.17 in $[\mathrm{CM}]$ works for Banach spaces, it now follows that every operator that is invertible on $H_{v_{p}}^{\infty}$ is also invertible on $H_{v_{p}, m}^{\infty}$. This can also be proved as Lemma 7 in [Z].

In connection with the above proof, recall that if $T$ is a bounded operator on a Banach space $X$ and $Y$ is an invariant closed subspace of $X$ under $T$, then $\sigma_{Y}\left(T_{\mid Y}\right)$ is not always contained in $\sigma_{X}(T)$. For example, if $T$ is the right shift on $l_{2}(\mathbb{Z})$ then $\sigma(T)$ is the unit circle although the spectrum of $\left.T\right|_{l_{2}(\mathbb{N})}$ is the closed disc.

Recall that $\left(z_{k}\right)$ is an iteration sequence for $\phi$ if $\phi\left(z_{k}\right)=z_{k+1}$ for all $k$. In order to follow the method of proof by Cowen and MacCluer [CM1] we need the next lemma. It should be pointed out that this approach of Cowen and MacCluer [CM1] was influenced by the work of Kamowitz in [K1]. 
Lemma 5 ([CM1] Lemma 13, Lemma 14). If $\phi$ is not an automorphism and $\phi(0)=0$, then given $0<r<1$, there exists $1 \leq M<\infty$ such that if $\left(z_{k}\right)_{k=-K}^{\infty}$ is an iteration sequence with $\left|z_{n}\right| \geq r$ for some non-negative integer $n$ and $\left(w_{k}\right)_{k=-K}^{n}$ are arbitrary numbers, then there exists $f \in H^{\infty}$ with $f\left(z_{k}\right)=w_{k},-K \leq k \leq n$ and $\|f\|_{\infty} \leq M \sup \left\{\left|w_{k}\right|:-K \leq k \leq n\right\}$. Further there exists $b<1$ such that for any iteration sequence $\left(z_{k}\right)$ we have $\left|z_{k+1}\right| /\left|z_{k}\right| \leq b$ whenever $\left|z_{k}\right| \leq 1 / 2$.

Lemma 6. Let $p>0$ and suppose that $\phi(0)=0$ and that $C_{\psi, \phi}$ is bounded on $H_{v_{p}}^{\infty}$. Then $\left\{\psi(0) \phi^{\prime}(0)^{n}\right\}_{n=0}^{\infty} \subset \sigma_{H_{v_{p}}^{\infty}}\left(C_{\psi, \phi}\right)$ and if $\lambda \neq 0$ is an eigenvalue of $C_{\psi, \phi}$, then $\lambda \in\left\{\psi(0) \phi^{\prime}(0)^{n}\right\}_{n=0}^{\infty}$.

Proof. Because $H_{v_{p}}^{\infty}$ contains the polynomials, the first statement follows as in $[\mathrm{K}]$, Lemma 2.3. The second result can be proved as Lemma 2.4 in [K].

Theorem 7. Let $p>0$ and suppose $\phi$, not an automorphism, has fixed point $a \in D$ and that $C_{\psi, \phi}: H_{v_{p}}^{\infty} \rightarrow H_{v_{p}}^{\infty}$ is bounded. Then

$$
\sigma_{H_{v_{p}}^{\infty}}\left(C_{\psi, \phi}\right)=\left\{\lambda \in \mathbb{C}:|\lambda| \leq r_{e, H_{v_{p}}^{\infty}}\left(C_{\psi, \phi}\right)\right\} \cup\left\{\psi(a) \phi^{\prime}(a)^{n}\right\}_{n=0}^{\infty}
$$

Proof. We can assume that $a=0$. Indeed, if $a \neq 0$ let $\phi_{a}(z)=\frac{a-z}{1-\bar{a} z}, \psi_{1}=\psi \circ \phi_{a}$ and $\phi_{1}=\phi_{a} \circ \phi \circ \phi_{a}$. Then $\phi_{1}(0)=0, \psi_{1}(0)=\psi(a), \phi_{1}^{\prime}(0)=\phi^{\prime}(a)$ and

$$
C_{\phi_{a}} \circ C_{\psi_{1}, \phi_{1}} \circ C_{\phi_{a}}^{-1}=C_{\psi, \phi}
$$

Hence $C_{\psi, \phi}$ and $C_{\psi_{1}, \phi_{1}}$ are similar. Therefore they have the same spectrum and the same essential spectral radius.

By Lemma 6 we have that $\left\{\psi(0) \phi^{\prime}(0)^{n}\right\}_{n=0}^{\infty} \subset \sigma_{H_{v_{p}}^{\infty}}\left(C_{\psi, \phi}\right)$. For $\lambda \in \sigma_{H_{v_{p}}^{\infty}}\left(C_{\psi, \phi}\right)$ and $|\lambda|>r_{e, H_{v_{p}}^{\infty}}\left(C_{\psi, \phi}\right)$, it follows that $\lambda$ is an eigenvalue (this is true for all bounded operators, see e.g. Proposition 2.2 in [BS]). If $\lambda \neq 0$ is an eigenvalue of $C_{\psi, \phi}$, then Lemma 6 gives that $\lambda=\psi(0) \phi^{\prime}(0)^{n}$ for some $n$, so it remains to show that

$$
\left\{\lambda \in \mathbb{C}:|\lambda| \leq r_{e, H_{v_{p}}^{\infty}}\left(C_{\psi, \phi}\right)\right\} \subset \sigma_{H_{v_{p}}^{\infty}}\left(C_{\psi, \phi}\right) .
$$

If $r_{e, H_{v_{p}}^{\infty}}\left(C_{\psi, \phi}\right)=0$, we are done since $0 \in \sigma_{H_{v_{p}}^{\infty}}\left(C_{\psi, \phi}\right)$ when $\phi$ is not an automorphism. We assume that $\rho:=r_{e, H_{v_{p}}^{\infty}}\left(C_{\psi, \phi}\right)>0$. Because the spectrum of $C_{\psi, \phi}$ is closed, we can choose $\lambda$ with $0<|\lambda|<\rho$. By Lemma 4 it is enough to show that $\lambda \in \sigma_{H_{v_{p}, m}^{\infty}}\left(C_{\psi, \phi}\right)$ for some $m$. Let $C_{m}$ denote the restriction of $C_{\psi, \phi}$ to the invariant closed subspace $H_{v_{p}, m}^{\infty}$. Since $C_{m}-\lambda I$ is not invertible if $\left(C_{m}-\lambda I\right)^{*}$ is not bounded from below, we just need to find $m$ with $\left(C_{m}-\lambda I\right)^{*}$ not bounded from below.

Let $1 \leq M<\infty$ be the constant in Lemma 5 for $r=1 / 4$. Iteration sequences will be denoted by $\left(z_{k}\right)_{k=-K}^{\infty}$ with $K>0$ and $\left|z_{0}\right| \geq 1 / 2$. Let $n:=\max \left\{k:\left|z_{k}\right| \geq 1 / 4\right\}$. Then $n \geq 0$ and $\left|z_{k}\right|<1 / 4$ for $k>n$. By Lemma 5 there also exists a $b<1$ such that $\left|z_{k+1} / z_{k}\right| \leq b$ for all $k \geq n$. We may assume that $1 / 2 \leq b<1$. It follows that $\left|z_{k}\right| \leq b^{k-n}\left|z_{n}\right|$ whenever $k \geq n$.

Since $\psi \in H(D)$ is continuous, $0<C:=\max \left\{\sup _{|z| \leq 1 / 4}|\psi(z)|,\left|\psi\left(z_{n}\right)\right|\right\}<\infty$. 
For fixed $p>0$ we now choose $m$ so large that

$$
\frac{b^{m} C}{|\lambda|}<\frac{9^{p}}{2 M 16^{p} 4^{p}}<1 / 2
$$

Given any iteration sequence $\left(z_{k}\right)_{k=-K}^{\infty}$ let us define the linear functional $L_{\lambda, \psi}$ on $H_{v_{p}, m}^{\infty}$ by

$$
L_{\lambda, \psi}(f)=\sum_{k=-K}^{\infty} \lambda^{-k} \psi\left(z_{-K}\right) \ldots \psi\left(z_{k-1}\right) f\left(z_{k}\right)
$$

where we agree that $\psi\left(z_{-K}\right) \ldots \psi\left(z_{-K-1}\right)=1$ in the first term of the sum. Then $L_{\lambda, \psi}$ is bounded since, with $n$ defined as above, Proposition 1 gives

$$
\begin{gathered}
\left|\sum_{k=-K}^{\infty} \lambda^{-k} \psi\left(z_{-K}\right) \ldots \psi\left(z_{k-1}\right) f\left(z_{k}\right)\right| \leq \\
c(m, p)\|f\|_{v_{p}}\left\{\sum_{k=-K}^{n}|\lambda|^{-k}\left|\psi\left(z_{-K}\right)\right| \ldots\left|\psi\left(z_{k-1}\right)\right|\left|z_{k}\right|^{m}\left(1-\left|z_{k}\right|^{2}\right)^{-p}\right. \\
\left.+\left|\psi\left(z_{-K}\right)\right| \ldots\left|\psi\left(z_{n-1}\right)\right| \sum_{k=n+1}^{\infty}|\lambda|^{-k}\left|\psi\left(z_{n}\right)\right| \ldots\left|\psi\left(z_{k-1}\right)\right|\left|z_{k}\right|^{m}\left(1-\left|z_{k}\right|^{2}\right)^{-p}\right\} .
\end{gathered}
$$

Since $\left|z_{k}\right|<1 / 4$ for $k>n,\left|z_{k}\right| \leq\left|z_{n}\right| b^{k-n}$ for $k \geq n$ and $b^{m} C /|\lambda|<1$, we get that the second sum

$$
\begin{gathered}
\leq\left|\psi\left(z_{-K}\right)\right| \ldots\left|\psi\left(z_{n-1}\right)\right| \sum_{k=n+1}^{\infty}|\lambda|^{-k} C^{k-n}\left|z_{k}\right|^{m}\left(\frac{16}{15}\right)^{p} \\
\leq\left|\psi\left(z_{-K}\right)\right| \ldots\left|\psi\left(z_{n-1}\right)\right|\left(\frac{16}{15}\right)^{p} \frac{\left|z_{n}\right|^{m}}{|\lambda|^{n}} \sum_{k=n+1}^{\infty}\left(\frac{b^{m} C}{|\lambda|}\right)^{k-n}<\infty .
\end{gathered}
$$

To show that $C_{m}^{*}-\lambda I$ is not bounded from below, we need to estimate

$$
\left\|\left(C_{m}^{*}-\lambda I\right) L_{\lambda, \psi}\right\|_{v_{p}, m} /\left\|L_{\lambda, \psi}\right\|_{v_{p}, m} .
$$

First notice that

$$
\left(C_{m}^{*}-\lambda I\right) L_{\lambda, \psi}=-\lambda^{K+1} \delta_{z_{-K}} .
$$

Now we find a lower bound for $\left\|L_{\lambda, \psi}\right\|_{v_{p}, m}$. For any iteration sequence $\left(z_{k}\right)_{k=-K}^{\infty}$ we get that there is $f \in H^{\infty},\|f\|_{\infty} \leq M$, satisfying

(i) $\left|f\left(z_{k}\right)\right|=1$ for $k=0$ and $k=n$,

(ii) $\frac{\psi\left(z_{-K}\right) \ldots \psi\left(z_{k-1}\right) z_{k}^{m} f\left(z_{k}\right)}{\lambda^{k}\left(1-\bar{z}_{0} z_{k}\right)^{2 p}} \geq 0$ for $k=0$ and $k=n$,

(iii) $f\left(z_{k}\right)=0$ for $-K \leq k<n, k \neq 0$.

(Later we will find that the iteration sequences $\left(z_{k}\right)_{k=-K}^{\infty}$ that we will use are such that $\left|\psi\left(z_{-K}\right)\right| \ldots\left|\psi\left(z_{-1}\right)\right|>0$ and therefore the expression in (ii) is strictly positive for $k=0$.) For such $f$ we calculate

$$
L_{\lambda, \psi}\left(\frac{z^{m} f(z)\left(1-\left|z_{0}\right|^{2}\right)^{p}}{\left(1-\bar{z}_{0} z\right)^{2 p}}\right)=\sum_{k=-K}^{\infty} \lambda^{-k} \psi\left(z_{-K}\right) \ldots \psi\left(z_{k-1}\right) z_{k}^{m} f\left(z_{k}\right) \frac{\left(1-\left|z_{0}\right|^{2}\right)^{p}}{\left(1-\bar{z}_{0} z_{k}\right)^{2 p}} .
$$


Since

$$
\frac{\left(1-\left|z_{0}\right|^{2}\right)^{p}\left(1-|z|^{2}\right)^{p}}{\left|1-\bar{z}_{0} z\right|^{2 p}}=\left(1-\left|\frac{z-\bar{z}_{0}}{1-\bar{z}_{0} z}\right|^{2}\right)^{p} \leq 1,
$$

we conclude that $g(z):=z^{m} f(z) \frac{\left(1-\left|z_{0}\right|^{2}\right)^{p}}{\left(1-\bar{z}_{0} z\right)^{2 p}}$ belongs to $H_{v_{p}, m}^{\infty}$ with norm smaller than $M$. Now we get

$$
\begin{gathered}
\left|\sum_{k=-K}^{\infty} \lambda^{-k} \psi\left(z_{-K}\right) \ldots \psi\left(z_{k-1}\right) g\left(z_{k}\right)\right| \geq \\
\frac{\left|\psi\left(z_{-K}\right)\right| \ldots\left|\psi\left(z_{-1}\right)\right|\left|z_{0}\right|^{m}}{\left(1-\left|z_{0}\right|^{2}\right)^{p}}+|\lambda|^{-n}\left|\psi\left(z_{-K}\right)\right| \ldots\left|\psi\left(z_{n-1}\right)\right|\left|z_{n}\right|^{m} \mid \frac{\left(1-\left|z_{0}\right|^{2}\right)^{p}}{\left|1-\bar{z}_{0} z_{n}\right|^{2 p}} \\
-\left|\sum_{k=n+1}^{\infty} \lambda^{-k} \psi\left(z_{-K}\right) \ldots \psi\left(z_{k-1}\right) g\left(z_{k}\right)\right|:=I+I I-I I I .
\end{gathered}
$$

Thus

$$
I I \geq \frac{\left|\psi\left(z_{-K}\right)\right| \ldots\left|\psi\left(z_{n-1}\right)\right|\left|z_{n}\right|^{m}\left(1-\left|z_{0}\right|^{2}\right)^{p}}{|\lambda|^{n} 4^{p}}
$$

and

$$
I I I \leq\left|\psi\left(z_{-K}\right)\right| \ldots\left|\psi\left(z_{n-1}\right)\right| M\left(\frac{16}{9}\right)^{p} \frac{\left|z_{n}\right|^{m}}{|\lambda|^{n}} \sum_{k=n+1}^{\infty}\left(\frac{b^{m} C}{|\lambda|}\right)^{k-n}\left(1-\left|z_{0}\right|^{2}\right)^{p} .
$$

Since

$$
\sum_{k=n+1}^{\infty}\left(\frac{b^{m} C}{|\lambda|}\right)^{k-n}<\frac{\frac{9^{p}}{2 M 16^{p} 4^{p}}}{1-\frac{9^{p}}{2 M 16^{p} 4^{p}}}<\frac{9^{p}}{M 16^{p} 4^{p}}
$$

we obtain that

$$
\left|L_{\lambda, \psi}(g)\right| \geq\left|\psi\left(z_{-K}\right)\right| \ldots\left|\psi\left(z_{-1}\right)\right| \frac{\left|z_{0}\right|^{m}}{\left(1-\left|z_{0}\right|^{2}\right)^{p}} .
$$

Consequently by Proposition 1,

$$
\left\|L_{\lambda, \psi}\right\|_{v_{p}, m} \geq \frac{1}{M} \frac{\left|\psi\left(z_{-K}\right)\right| \ldots\left|\psi\left(z_{-1}\right)\right|\left|z_{0}\right|^{m}}{\left(1-\left|z_{0}\right|^{2}\right)^{p}} \geq \frac{\left|\psi\left(z_{-K}\right)\right| \ldots\left|\psi\left(z_{-1}\right)\right|}{M c(m, p)}\left\|\delta_{z_{0}}\right\|_{v_{p}, m} .
$$

Recall that

$$
\rho=r_{e, H_{v_{p}}^{\infty}}\left(C_{\psi, \phi}\right)=\lim _{n}\left\|C_{\psi, \phi}^{n}\right\|_{e, v_{p}}^{\frac{1}{n}}
$$

and

$$
C_{\psi, \phi}^{n} f(w)=\psi(w) \ldots \psi\left(\phi_{n-1}(w)\right) C_{\phi_{n}} f(w), \quad w \in D, f \in H_{v_{p}}^{\infty},
$$

where $\phi_{n}=\phi \circ \ldots \circ \phi$ (n times). Hence $C_{\psi, \phi}^{n}: H_{v_{p}}^{\infty} \rightarrow H_{v_{p}}^{\infty}$ is a bounded weighted composition operator and we get by Theorem 4.2 in [CHD] or Theorem 2.1 in [MR] that

$$
\left\|C_{\psi, \phi}^{n}\right\|_{e, v_{p}}=\lim _{r \rightarrow 1} \sup _{\left|\phi_{n}(w)\right|>r} \frac{|\psi(w)| \ldots\left|\psi\left(\phi_{n-1}(w)\right)\right|\left(1-|w|^{2}\right)^{p}}{\left(1-\left|\phi_{n}(w)\right|^{2}\right)^{p}} .
$$

Given $0<|\lambda|<\rho$ we can pick $\mu$ so that $|\lambda|<\mu<\rho$. Thus there exists $n_{0}$ such that for all $n \geq n_{0}$,

$$
\left\|C_{\psi, \phi}^{n}\right\|_{e, v_{p}}>\mu^{n}
$$


Hence for any $K \geq n_{0}$ we can find a $w \in D$ so that

(i) $|\psi(w)| \ldots\left|\psi\left(\phi_{K-1}(w)\right)\right|\left(\frac{1-|w|^{2}}{1-\left|\phi_{K}(w)\right|^{2}}\right)^{p} \geq \mu^{K}>0$,

(ii) $\left|\phi_{K}(w)\right| \geq 1 / 2$,

(iii) $\frac{\left\|\delta_{\phi_{K}(w)}\right\|_{v_{p}, m}}{\left\|\delta_{w}\right\| \|_{v_{p}, m}} \geq \frac{1}{2^{m}} \frac{\left\|\delta_{\phi_{K}(w)}\right\|_{v_{p}}}{\left\|\delta_{w}\right\|_{v_{p}}}=\frac{1}{2^{m}} \frac{\left(1-|w|^{2}\right)^{p}}{\left(1-\left|\phi_{K}(w)\right|^{2}\right)^{p}}$.

In (iii) we have used Lemma 3 and a remark in the introduction.

Thus

$$
\frac{\left\|\delta_{\phi_{K}(w)} \mid\right\|_{v_{p}, m}}{\left\|\delta_{w}\right\|_{v_{p}, m}} \geq \frac{\mu^{K}}{2^{m}|\psi(w)| \ldots\left|\psi\left(\phi_{K-1}(w)\right)\right|} .
$$

This means that for every $K \geq n_{0}$ with the above choice of $w \in D$ we can form an iteration sequence $\left(z_{k}\right)_{k=-K}^{\infty}$ by letting $z_{-K}=w$ and $z_{k+1}=\phi\left(z_{k}\right)$ for $k \geq-K$. Then $\left|z_{0}\right|=\left|\phi_{K}(w)\right| \geq 1 / 2$.

Now we get

$$
\begin{aligned}
\frac{\left\|\left(C_{m}^{*}-\lambda I\right) L_{\lambda, \psi}\right\|_{v_{p}, m}}{\left\|L_{\lambda, \psi}\right\| \|_{p}, m} & \leq \\
\frac{M c(m, p)}{\left|\psi\left(z_{-K}\right)\right| \ldots\left|\psi\left(z_{-1}\right)\right|\left\|\delta_{z_{0}}\right\|_{v_{p}, m}}|\lambda|^{K+1}|| \delta_{z_{-K}} \|_{v_{p}, m} & \leq|\lambda| M c(m, p) 2^{m}\left(\frac{|\lambda|}{\mu}\right)^{K} .
\end{aligned}
$$

By choosing $K \geq n_{0}$ large enough, we see that $C_{m}^{*}-\lambda I$ is not bounded from below.

When $C_{\psi, \phi}: H_{v_{p}}^{0} \rightarrow H_{v_{p}}^{0}$ is bounded, we get that $C_{\psi, \phi}=C_{\psi, \phi}^{* *}: H_{v_{p}}^{\infty} \rightarrow H_{v_{p}}^{\infty}$ is also bounded and we can apply Theorem 7 . Further, $C_{\psi, \phi}^{n}$ is bounded on both $H_{v_{p}}^{0}$ and $H_{v_{p}}^{\infty}$ and $C_{\psi, \phi}^{n} f(w)=\psi(w) \ldots \psi\left(\phi_{n-1}(w)\right) C_{\phi_{n}} f(w)$. Therefore $C_{\psi, \phi}^{n}$ is a bounded weighted composition operator on both $H_{v_{p}}^{0}$ and $H_{v_{p}}^{\infty}$. Hence using Theorem 2.2 and its proof in [MR] we obtain that the essential norm of $C_{\psi, \phi}^{n}$ on $H_{v_{p}}^{0}$ coincides with the essential norm of $C_{\psi, \phi}^{n}$ on $H_{v_{p}}^{\infty}$ when $C_{\psi, \phi}$ is bounded on $H_{v_{p}}^{0}$. Thus $r_{e, H_{v_{p}}^{0}}\left(C_{\psi, \phi}\right)=r_{e, H_{v_{p}}^{\infty}}\left(C_{\psi, \phi}\right)$ and we can formulate the following result.

Corollary 8. Suppose that $\phi$, not an automorphism, has fixed point $a \in D, p>0$ and that $C_{\psi, \phi}: H_{v_{p}}^{0} \rightarrow H_{v_{p}}^{0}$ is bounded. Then

$$
\sigma_{H_{v_{p}}^{0}}\left(C_{\psi, \phi}\right)=\left\{\lambda \in \mathbb{C}:|\lambda| \leq r_{e, H_{v_{p}}^{0}}\left(C_{\psi, \phi}\right)\right\} \cup\left\{\psi(a) \phi^{\prime}(a)^{n}\right\}_{n=0}^{\infty} .
$$

We shall now apply Theorem 7 to composition operators on Bloch type spaces. Therefore we will pay attention to weighted composition operators $C_{\psi, \phi}$ with $\psi=$ $\phi^{\prime}$.

Let $\mathcal{B}_{p}, 0<p<\infty$, denote the Bloch type spaces of functions $f \in H(D)$ satisfying $f(0)=0$ and ||$f||_{\mathcal{B}_{p}}:=\sup _{z \in D}\left(1-|z|^{2}\right)^{p}\left|f^{\prime}(z)\right|<\infty$. The space $\mathcal{B}_{p}$ is a Banach space under the norm $\|\cdot\|_{\mathcal{B}_{p}}$. The map $S_{p}: \mathcal{B}_{p} \rightarrow H_{v_{p}}^{\infty}$ given by $S_{p}(f)=f^{\prime}$ is an onto isometry.

Assume that $\phi(0)=0$ and that $C_{\phi}$ is bounded on $\mathcal{B}_{p}$. Then we have that $C_{\phi}=S_{p}^{-1} \circ C_{\phi^{\prime}, \phi} \circ S_{p}$ and therefore

$$
\sigma_{\mathcal{B}_{p}}\left(C_{\phi}\right)=\sigma_{H_{v_{p}}^{\infty}}\left(C_{\phi^{\prime}, \phi}\right) \text { and } r_{e, \mathcal{B}_{p}}\left(C_{\phi}\right)=r_{e, H_{v_{p}}^{\infty}}\left(C_{\phi^{\prime}, \phi}\right) .
$$

Thus we conclude the next result from Theorem 7 . 
Corollary 9. Suppose that $\phi$, not an automorphism, fixes the origin. If the composition operator $C_{\phi}: \mathcal{B}_{p} \rightarrow \mathcal{B}_{p}, 0<p<\infty$, is bounded, then

$$
\sigma_{\mathcal{B}_{p}}\left(C_{\phi}\right)=\left\{\lambda \in \mathbb{C}:|\lambda| \leq r_{e, \mathcal{B}_{p}}\left(C_{\phi}\right)\right\} \cup\left\{\phi^{\prime}(0)^{n}\right\}_{n=1}^{\infty}
$$

(For $p \geq 1$, the boundedness assumption on $C_{\phi}$ always holds.)

We remark that a similar result also holds for little Bloch type spaces.

In [MS] MacCluer and Saxe made the following Conjecture: Let $\phi$ be a univalent self map on $D$, not an automorphism, with fixed point in $D$, and such that $r_{e, H^{2}}\left(C_{\phi}\right) \neq 0$. Then $\sigma_{\mathcal{B}}\left(C_{\phi}\right)=\bar{D}$, where $\mathcal{B}$ is the classical Bloch space with the norm $\|f\|_{\mathcal{B}}:=|f(0)|+\sup _{z \in D}\left(1-|z|^{2}\right)\left|f^{\prime}(z)\right|$.

In order to study this conjecture we can assume without loss of generality that $\phi(0)=0$. Therefore by the Schwarz-Pick lemma it follows that $\sigma_{\mathcal{B}}\left(C_{\phi}\right)$ is contained in the closed unit disk. Let $\mathcal{B}_{1} \oplus \mathbb{C}$ be endowed with the norm $\|(f, \alpha)\|=\|f\|_{\mathcal{B}_{1}}+|\alpha|$. Then the map $T_{1}: \mathcal{B}_{1} \oplus \mathbb{C} \rightarrow \mathcal{B}$ given by $T_{1}(f, \alpha)=\alpha+f$ is an onto isometry and satisfies $T_{1}^{-1} \circ C_{\phi} \circ T_{1}=\left(C_{\phi \mid \mathcal{B}_{1}}, i d\right)$. Consequently $\sigma_{\mathcal{B}}\left(C_{\phi}\right)=\sigma_{\mathcal{B}_{1}}\left(C_{\phi}\right) \cup\{1\}$. By the above we know that

$$
\left\|C_{\phi_{n}}\right\|_{e, \mathcal{B}_{1}}=\left\|C_{\phi^{\prime}, \phi}^{n}\right\|_{e, v_{1}}=\lim _{r \rightarrow 1} \sup _{\left|\phi_{n}(w)\right|>r} \frac{\left|\left(\phi_{n}\right)^{\prime}(w)\right|\left(1-|w|^{2}\right)}{1-\left|\phi_{n}(w)\right|^{2}} .
$$

Since $r_{e, H^{2}}\left(C_{\phi}\right) \neq 0$ and $\phi$ is univalent, every $\phi_{n}$ has finite angular derivative at some point of $\partial D$ [Sh], p. 57. The Schwarz-Pick lemma gives that $\left\|C_{\phi_{n}}\right\|_{e, \mathcal{B}_{1}} \leq 1$. Now $\phi_{n}$ has finite angular derivative at some point $\xi \in \partial D$ and we can apply the Julia-Caratheodory theorem $[\mathrm{Sh}]$ to get

$$
1=\liminf _{w \rightarrow \xi} \frac{\left|\left(\phi_{n}\right)^{\prime}(w)\right|\left(1-|w|^{2}\right)}{1-\left|\phi_{n}(w)\right|^{2}} \leq \lim _{r \rightarrow 1} \sup _{\left|\phi_{n}(w)\right|>r} \frac{\left|\left(\phi_{n}\right)^{\prime}(w)\right|\left(1-|w|^{2}\right)}{1-\left|\phi_{n}(w)\right|^{2}}
$$

See [MR1] for a similar argument. Therefore $\left\|C_{\phi_{n}}\right\|_{e, \mathcal{B}_{1}}=1$ and consequently we obtain that $r_{e, \mathcal{B}_{1}}\left(C_{\phi}\right)=1$. Thus we have proved the conjecture of MacCluer and Saxe in $[\mathrm{MS}]$.

Corollary 10. Suppose that $\phi$ is univalent, not an automorphism, fixes a point in D. If $r_{e, H^{2}}\left(C_{\phi}\right) \neq 0$, then $\sigma_{\mathcal{B}}\left(C_{\phi}\right)=\bar{D}$.

In [MS] MacCluer and Saxe showed that $r_{e, H^{2}}\left(C_{\phi}\right)=s^{1 / 2}$ for $\phi(z)=\frac{s z}{1-(1-s) z}$, $0<s<1$. The function $\phi$ is univalent, not an automorphism and fixes zero. For any $n \in \mathbb{N}$,

$$
\phi_{n}(z)=\frac{s^{n} z}{1-\left(1-s^{n}\right) z}
$$

and

$$
\left|\left(\phi_{n}\right)^{\prime}(z)\right|=\frac{s^{n}}{\left|1-\left(1-s^{n}\right) z\right|^{2}}=\frac{\left|\phi_{n}(z)\right|^{2}}{s^{n}|z|^{2}} .
$$

By Schwarz lemma, $C_{\phi}: \mathcal{B}_{p} \rightarrow \mathcal{B}_{p}$ is bounded for all $p>0$. MacCluer and Saxe [MS] point out that for every $n \in \mathbb{N}$, the angular derivative of $\phi_{n}$ at 1 is given by 
$\left(\phi_{n}\right)^{\prime}(1)=1 / s^{n}$ and at every other $\xi \in \partial D$ the angular derivative is infinite. Hence, by the proof of Theorem 2.2 in [MR] and Proposition 2.46 in [CM],

$$
\lim _{r \rightarrow 1} \sup _{\left|\phi_{n}(z)\right|>r}\left(\frac{1-|z|}{1-\left|\phi_{n}(z)\right|}\right)^{p}=\limsup _{|z| \rightarrow 1}\left(\frac{1-|z|}{1-\left|\phi_{n}(z)\right|}\right)^{p}=s^{n p} .
$$

Therefore

$$
\left\|C_{\phi_{n}}\right\|_{e, \mathcal{B}_{p}}=\lim _{r \rightarrow 1} \sup _{\left|\phi_{n}(z)\right|>r} \frac{\left|\phi_{n}(z)\right|^{2}}{s^{n}|z|^{2}}\left(\frac{1-|z|^{2}}{1-\left|\phi_{n}(z)\right|^{2}}\right)^{p}=\frac{1}{s^{n}} s^{n p}=s^{n(p-1)} .
$$

Thus we get that $r_{e, \mathcal{B}_{p}}\left(C_{\phi}\right)=s^{p-1}$ and

$$
\sigma_{\mathcal{B}_{p}}\left(C_{\phi}\right)=\left\{\lambda \in \mathbb{C}:|\lambda| \leq s^{p-1}\right\} \cup\left\{s^{n}\right\}_{n=1}^{\infty} .
$$

For $p=1$ this follows of course from Corollary 10 and $r_{e, \mathcal{B}_{p}}\left(C_{\phi}\right) \rightarrow 0$ when $p \rightarrow \infty$.

Acknowledgement. The authors are very indebted to Pablo Galindo for discussions on the subject of the paper.

\section{REFERENCES}

[BBT] K. D. Bierstedt, J. Bonet, J. Taskinen, Associated weights and spaces of holomorphic functions, Studia Math. 127 (1998), 137-168.

[BDLT] J. Bonet, P. Domański, M. Lindström, J. Taskinen, Composition operators between weighted Banach spaces of analytic functions, J. Austral. Math. Soc. (Series A) 64 (1998), 101-118.

[BDL] J. Bonet, P. Domański, M. Lindström, Essential norm and weak compactness of composition operators on weighted Banach spaces of analytic functions, Canad. Math. Bull. 42 (1999), 139-148.

[BS] P. Bourdon, J. Shapiro, Mean growth of Koenigs eigenfunctions, J. Amer. Math. Soc. 10 (1997), 299-325.

[CHD] M.D. Contreras, A.G. Hernandez-Díaz, Weighted composition operators in weighted Banach spaces of analytic functions, J. Austral. Math. Soc. (Series A) 69 (2000), 4160 .

[CM] C. Cowen, B. MacCluer, Composition Operators on Spaces of Analytic Functions, CRC Press, Boca Raton, 1995.

[CM1] C. Cowen, B. MacCluer, Spectra of some composition operators, J. Functional Analysis 125 (1994), 223-251.

[K] H. Kamowitz, Compact operators of the form $u C_{\varphi}$, Pacific J. Math. 80 (1979), 205-211.

[K1] H. Kamowitz, The spectra of some composition operators, J. Functional Analysis 18 (1975), 132-150.

[MS] B. MacCluer, K. Saxe, Spectra of composition operators on the Bloch and Bergman spaces, Isr. J. Math. 128 (2002), 325-354.

[MR] A. Montes-Rodríguez, Weighted composition operators on weighted Banach spaces of analytic functions, J. London Math. Soc. 61 (2000), 872-884.

[MR1] A. Montes-Rodríguez, The essential norm of a composition operator on Bloch spaces, Pacific J. Math. 188 (1999), 339-351.

[Sh] J.H. Shapiro, Composition Operators and Classical Function Theory, Springer, 1993. 
[SW] A. L. Shields, D. L. Williams, Bounded projections, duality, and multipliers in spaces of analytic functions, Trans. Amer. Math. Soc. 162 (1971), 287-302.

[Z] L. Zheng, The essential norms and spectra of composition operators on $H^{\infty}$, Pacific J. Math. 203 (2002), 503-510.

[W] P. Wojtaszczyk, Banach Spaces for Analysts, Cambridge Univ. Press, Cambridge, 1991.

Department of Mathematical Sciences, Kent State University, Ohio, 44242, USA

E-mail address: aron@mcs.kent.edu

Department of Mathematics, Abo Akademi University, Fin-20500 Abo, Finland

E-mail address: mikael.lindstrom@abo.fi 